\title{
I ntraoperative radiation therapy for high risk soft tissue sarcoma resection margins
}

\author{
J acqueline Oxenberg ${ }^{1}$, Harish K. Malhotra ${ }^{2}$, Kristopher Attwood ${ }^{3}$, J ohn M. Kane II I ${ }^{1}$, Kilian \\ Salerno ${ }^{2}$
}

1. Surgical Oncology, Roswell Park Cancer Institute, Buffalo, NY, USA. 2. Radiation Oncology, Roswell Park Cancer Institute, Buffalo, NY, USA. 3. Biostatistics, Roswell Park Cancer Institute, Buffalo, NY, USA

Correspondence: Jacqueline Oxenberg, DO. Address: Department of Surgical Oncology, Roswell Park Cancer Institute EIm \& Carlton Streets Buffalo, NY 14263, USA. Email: jackieofrg@yahoo.com

Received: October 30, 2014

Accepted: December 17, 2014 Online Published: December 23, 2014

DOI : $10.5430 /$ jst.v4n4p25

URL: http://dx.doi.org/10.5430/jst.v4n4p25

\section{Abstract}

Background and objectives: External beam radiation (EBRT) can reduce local recurrence (LR) of soft tissue sarcomas (STS). The addition of intraoperative radiation therapy (IORT) can deliver high dose radiation boost to anticipated "high risk" margins while sparing adjacent structures.

Methods: A retrospective review (2004-2012) was performed of STS treated with surgical resection and IORT using HDR brachytherapy for anticipated close/positive margins.

Results: Twenty-four patients underwent 25 resections with IORT (1 patient had 2 separate recurrences). Tumors were primary in $72 \%$, deep in $96 \%$ and intermediate/high grade in $84 \%$. Tumor locations were extremity (44\%), retroperitoneal (40\%), truncal (12\%) and neck (4\%). Common histologies included pleomorphic (32\%), liposarcoma (12\%) and myxofibrosarcoma (12\%). Neoadjuvantly, 3 received chemotherapy and 13 received EBRT (median 50Gy; range 45-54). Median IORT dose was 12Gy (range 10-17.5). Margins were microscopically positive in 20\%; none were grossly positive. Adjuvantly, 5 received EBRT (median 46Gy, range 45-50) and 2 received chemotherapy. Median follow-up was 20.1 months (range 2.7-96.4). No recurrences occurred at the IORT sites. Two-year LR free survival was $60.8 \%$ and disease specific survival was $84 \%$.

Conclusion: Use of IORT at time of STS resection was effective at preventing LR at the treated site despite "high risk" features.

\section{Key words}

Intraoperative radiation, Sarcoma, High risk, Margins, Brachytherapy, Intraoperative radiation therapy

\section{I ntroduction}

Soft tissue sarcomas (STS) can occur throughout the body, but most commonly occur in the extremities, trunk, and retroperitoneum. Surgical resection with pathologically negative margins remains the treatment of choice but local recurrences continue to be problematic. This is especially true when tumors lie in close proximity to critical, unresectable structures. Use of external beam radiation therapy (EBRT) either pre-operatively or post-operatively can improve local 
control ${ }^{[1-7]}$. Patients with tumors that are high grade, locally recurrent or at high risk for positive margins are at increased risk for local recurrence and distant metastases ${ }^{[3,6,8-11]}$.

Advances in radiation therapy allow for more conformal target coverage, yet normal tissue tolerances limit dose ${ }^{[6,12-26]}$. Intraoperative radiation therapy (IORT) techniques permit high dose delivery to areas at risk while sparing adjacent surrounding normal tissues. IORT boost doses to areas at risk for close or positive margins can be used to enhance local control (LC). As such, IORT is an appealing modality to improve local control by allowing delivery of high doses of radiation while minimizing the dose to adjacent normal tissues that would otherwise be located within external beam radiation fields. IORT allows for local delivery of radiation to a focused field and can be delivered in a single session. Only one randomized trial to date exists showing a significantly improved local control rate of $60 \%$ using a combination of 20 Gy IORT and 35-40 Gy EBRT compared to 20\% with 50-55 Gy postoperative EBRT alone ${ }^{[16]}$. Other reported studies showed intraoperative radiation therapy (IORT) for extremity, retroperitoneal and truncal STS provided excellent local control, with limited acute toxicities ${ }^{[15,16,18,19,21-26]}$. With the benefit of additional radiation therapy to focal high-risk areas while sparing surrounding structures, we hypothesized that use of IORT would decrease local recurrence rates and improve local disease control.

\section{Methods}

\subsection{Data collection}

Institutional Review Board approval was obtained and a retrospective review of all STS was performed. Our institution's cancer registry database was queried to identify patients for evaluation who had IORT at time of definitive surgery for STS. Patients were included if they underwent surgical resection and IORT treatments at our institution. Patients were excluded if they did not undergo IORT at time of resection or if they had uterine or genitourinary based sarcomas. Chart based review was performed for data collection.

\subsection{Treatment}

In general, it is our institutional practice to consider preoperative radiation for patients at high risk for local recurrence. This includes tumors that are large, high grade, or in close proximity to critical and/or unresectable structures. All patients with STS are discussed and treated by a multidisciplinary team with expertise in sarcoma. Postoperative EBRT is used when it is unclear if radiation was needed prior to resection positive margins. This includes tumors at different locations but also allows patients with extremity tumors an attempt for limb salvage. IORT was used as a boost to address specific areas at risk for close or different locations. In patients with recurrent extremity tumors, IORT was used with re-resection in attempt for limb salvage.

All patients were evaluated preoperatively at our institution by the performing surgeons and radiation oncologists. Treatment plans were formulated accordingly for resection, additional radiation with external beam and systemic therapy. The areas of concern for close or positive margins were identified. All patients were treated with IORT at the time of surgical resection. Margins were considered positive if tumor was present at the inked margin.

\section{ORT HDR brachytherapy technique}

High dose rate (HDR) brachytherapy using an Iridium192 source was delivered using Freiburg flaps and delivered in a specially designed and shielded operating room. The customized applicators were designed to treat the operative bed and areas of concern as defined intra-operatively by the surgeon and radiation oncologist. Treatment plans were generated on either Plato ${ }^{\circledR}$ Treatment planning system or Oncentra ${ }^{\circledR}$ Treatment planning system utilizing the standard geometry available for the Freiburg applicator. MicroSelectron ${ }^{\circledR}$ high dose rate (HDR) unit (model digital V3, Manufacturer Elekta Inc., Stockhom, Sweden) was used for dose delivery. The number of catheters, active dwell positions along each catheter, prescription depth, and dose prescription were defined. In the majority of the cases, prescription depth was $5 \mathrm{~mm}$ from the 
surface of the flap (10 $\mathrm{mm}$ from the source center and $5 \mathrm{~mm}$ in tissue). Catheter dose points along all the active dwell positions at the prescription depth were defined. Dose point optimization was then carried out to ensure a uniform dose to all the dose points. The Freiburg flap and catheters were secured to the operative bed and normal surrounding tissues were shielded. Adjacent uninvolved normal tissues including ureters, nerves, vessels and skin were typically shielded with lead and held in place with retractors. The patient was monitored throughout treatment delivery. The flap and shielding were then removed and the remainder of the operative procedure completed.

Figure 1 shows HDR brachytherapy for a multiply recurrent well-differentiated right retroperitoneal liposarcoma, with areas of myxoid dedifferentiation. Recurrent tumor measured $4.3 \mathrm{~cm} \times 4 \mathrm{~cm} \times 3.3 \mathrm{~cm}$ and was located between the right external iliac vessels and bladder. Potentially close/microscopically margins were suspected at the right external iliac vessels. A customized Freiberg Flap was designed to treat the areas of concern overlying the surface of the vessels. Customized lead shielding was utilized to shield the bowel, bladder and ureter (wrapped in sterile impermeable draping and held in place with towels and retractors). These organs were displaced with retractors, shielded with lead shielding with wet lap packs. The skin surface was additionally shielded. A treatment plan was designed to treat $10 \mathrm{~Gy}$ to the surface of the Freiburg flap (5 mm from the source) using HDR brachytherapy using an Iridium 192 source.

Figure 1. HDR brachytherapy is used to treat a multiply recurrent well-differentiated right retroperitoneal liposarcoma, with areas of myxoid dedifferentiation. A customized Freiberg Flap was designed to treat the areas of concern overlying the surface of the vessels. Customized lead shielding was utilized to shield the bowel, bladder and ureter (wrapped in sterile impermeable draping and held in place with towels and retractors). These organs were displaced with retractors, shielded with lead shielding with wet lap packs. The skin surface was additionally shielded. A treatment plan was designed to treat 10 Gy to the surface of the Freiburg flap (5 $\mathrm{mm}$ from the source) using HDR brachytherapy using an Iridium 192 source.

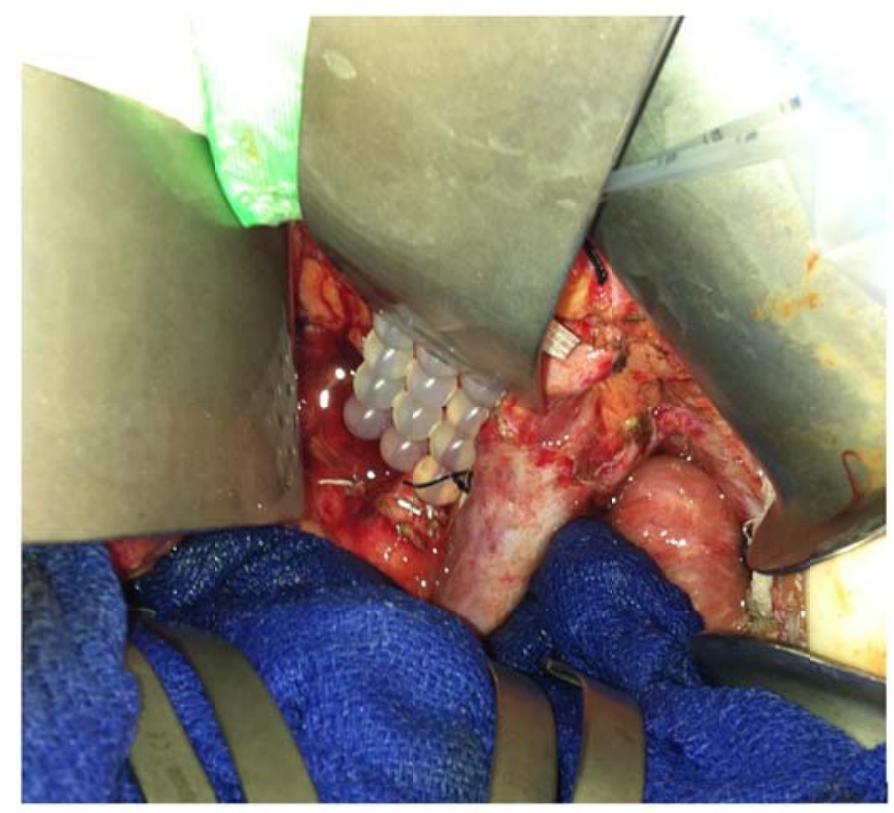

\subsection{Statistical analysis}

Statistical analyses were conducted per tumor treatment. Summary statistics for the entire sample were reported as means, medians, and standard deviations for continuous variables; and as frequencies and relative frequencies for categorical variables. The patient and treatment characteristics calculated included: locations, margin status, recurrent disease, and complication status/grade when available and/or appropriate. The Clavien-Dindo Classification was used to score postoperative wound complications ${ }^{[27]}$. Comparisons of patient and treatment characteristics between groups were assessed using the Wilcoxon's and Chi-square or Fisher's exact tests for continuous and categorical variables, respectively.

The time-to-event outcomes were summarized for the overall sample and by group using the standard Kaplan-Meier methods. Outcomes evaluated included: local recurrence (time from surgery until local recurrence, death or last follow-up; LR), local recurrence free survival (time from surgery until local recurrence, death, or last follow-up; LRFS), disease specific survival (time from surgery until death with/from disease, death from other cause or last follow-up; DSS), disease 
free survival (time from surgery until local/distant recurrence, death, or last follow-up; DFS), and distant disease free survival (time from surgery until distant recurrence, death, or last follow-up; DDFS). Comparisons of time-to-event outcomes between groups were assessed using the log-rank test.

All analyses were conducted in SAS v9.3 (Cary, NC) at a significance level of 0.05 .

\section{Results}

\subsection{Patient characteristics and prior treatment details}

Twenty-four patients and 25 tumors were treated with IORT at time of resection (1 patient for 2 separate recurrences). Median age at time of treatment was 61 years (range 19-81, SD=15.8). Patient, tumor and prior treatment characteristics can be seen in Tables 1 and 2. All extremity tumors were deep and 1 (33\%) truncal tumor was superficial. Most common subtypes included: 32\% pleomorphic, $12 \%$ liposarcoma, and 12\% myxofibrosarcoma. Prior treatment courses for the 7 patients with recurrent disease included prior definitive resections, 5 of which were performed at outside institutions. One patient received preoperative radiation, 1 received preoperative chemotherapy. One patient was treated with resection and IORT after recurring after resection and adjuvant radiation. The recurrence for this patient was local but not at the site of prior IORT. Prior adjuvant treatments included 3 tumors with radiation, 1 with chemotherapy, and 1 with chemotherapy and radiation.

Table 1. Overall Patient, Tumor and Treatment Characteristics

\begin{tabular}{|c|c|c|c|}
\hline Variables & Character & $\mathbf{N}$ & $\%$ \\
\hline Gender & Female & 14 & 66 \\
\hline \multirow{2}{*}{ Tumor Status } & Primary & 17 & 72 \\
\hline & Recurrent & 7 & 28 \\
\hline \multirow{7}{*}{ Location } & Retroperitoneal & 10 & 40 \\
\hline & Upper Extremity & 3 & 27 \\
\hline & Lower Extremity & 8 & 73 \\
\hline & Proximal Extremity & 6 & 55 \\
\hline & Distal Extremity & 5 & 45 \\
\hline & Trunk & 3 & 12 \\
\hline & Neck & 1 & 4 \\
\hline \multirow{2}{*}{ Depth } & Superficial & 1 & 4 \\
\hline & Deep & 24 & 96 \\
\hline \multirow{3}{*}{ Grade } & Low & 4 & 16 \\
\hline & Intermediate & 4 & 16 \\
\hline & High & 17 & 68 \\
\hline \multirow{3}{*}{ Median Size (cm) } & Extremity & 6.5 & \\
\hline & Retroperitoneal & 9.5 & \\
\hline & Trunk/Neck & 9 & \\
\hline History of RT & Any & 3 & 12 \\
\hline \multirow{2}{*}{ Prior Resection } & Any & 7 & 28 \\
\hline & Outside Institution & 5 & 20 \\
\hline \multirow{4}{*}{$\begin{array}{l}\text { Adjuvant Tx After Prior } \\
\text { Resection }\end{array}$} & Any & 5 & 20 \\
\hline & Chemo & 2 & 40 \\
\hline & $\mathrm{RT}$ & 2 & 40 \\
\hline & Both & 1 & 20 \\
\hline \multirow{3}{*}{ Neoadj Tx } & EBRT Only (Median 50Gy) & 13 & 52 \\
\hline & Chemo Only & 1 & 4 \\
\hline & ChemoRT & 1 & 4 \\
\hline
\end{tabular}

Note. $\mathrm{RT}=$ radiation therapy, $\mathrm{Tx}=$ treatment, chemo= chemotherapy, Neoadj= neoadjuvant, $\mathrm{EBRT}=$ external beam radiation therapy, ChemoRT= chemoradiation 
Table 2. Tumor Characteristics and Treatment Specifics

\begin{tabular}{|c|c|c|c|c|c|c|c|c|c|}
\hline Tumor & Location & $\begin{array}{l}\text { Age at Diagnosis } \\
\text { (years) }\end{array}$ & $\begin{array}{l}\text { Recurrent } \\
\text { Tumor }\end{array}$ & Histology & Grade & $\begin{array}{l}\text { Previous } \\
\text { Surgery }\end{array}$ & $\begin{array}{l}\text { Prior } \\
\text { RT }\end{array}$ & $\begin{array}{l}\text { Preop } \\
\text { RT }\end{array}$ & $\begin{array}{l}\text { Dose } \\
\text { (Gy) }\end{array}$ \\
\hline 1 & Anterior R lower leg & 61 & Yes & Myxofibrosarcoma & Interm & Yes & No & No & 0 \\
\hline 2 & $\begin{array}{l}\text { Anterior R lower leg, } \\
\text { over tibia }\end{array}$ & 61 & Yes & Myxofibrosarcoma & Interm & Yes & Yes & No & 0 \\
\hline 3 & Anterior L thigh & 35 & No & $\begin{array}{l}\text { Pleomorphic } \\
\text { leioyomyosarcoma }\end{array}$ & High & No & No & Yes & 50 \\
\hline 4 & $\begin{array}{l}\text { Anteromedial R lower } \\
\text { leg }\end{array}$ & 78 & No & Pleomorphic sarcoma & High & No & No & Yes & 50 \\
\hline 5 & Medial gastrocnemius & 61 & No & Myxofibrosarcoma & Low & No & No & Yes & 50 \\
\hline 6 & Anterolateral R leg & 59 & No & Myxoid liposarcoma & Interm & No & No & Yes & 50 \\
\hline 7 & $\mathrm{R}$ pelvic sidewall & 65 & Yes & $\begin{array}{l}\text { Epithelioid } \\
\text { hemangioendothelioma }\end{array}$ & High & Yes & No & Yes & 45 \\
\hline 8 & Anterior R thigh & 65 & No & Leiomyosarcoma & High & No & No & No & 0 \\
\hline 9 & Proximal L thigh & 80 & No & Pleomorphic sarcoma & High & No & No & Yes & 54 \\
\hline 10 & R paraspinal & 48 & Yes & $\begin{array}{l}\text { Myxoid } \\
\text { pleomorphic/leiomyosa } \\
\text { rcoma }\end{array}$ & High & Yes & No & No & 0 \\
\hline 11 & Left retroperitoneal & 60 & No & Myxofibrosarcoma & Low & No & No & Yes & 45 \\
\hline 12 & Left retroperitoneal & 58 & Yes & Myxoid NOS & High & Yes & No & Yes & 53 \\
\hline 13 & L psoas & 19 & No & Alveolar soft part & High & No & No & Yes & 50 \\
\hline 14 & L paraspinal thoracic & 61 & No & $\begin{array}{l}\text { Monophasic synovial } \\
\text { cell }\end{array}$ & High & No & No & No & 0 \\
\hline 15 & $\mathrm{R}$ lateral breast & 56 & No & $\begin{array}{l}\text { Pleomorphic spindle } \\
\text { cell }\end{array}$ & High & No & Yes & No & 0 \\
\hline 16 & $\mathrm{~L}$ retroperitoneum & 56 & No & $\begin{array}{l}\text { Pleomorphic } \\
\text { leiomyosarcoma }\end{array}$ & High & No & No & No & 0 \\
\hline 17 & L retroperitoneum & 24 & Yes & Periph nerve sheath & High & No & No & No & 0 \\
\hline 18 & $\mathrm{R}$ retroperitoneum & 57 & No & Leiomyosarcoma & Interm & No & No & No & 0 \\
\hline 19 & L upper posterior arm & 50 & No & Hemangiopericytoma & High & No & No & Yes & 50 \\
\hline 20 & $\mathrm{~L}$ axilla & 60 & No & Pleomorphic sarcoma & High & No & No & Yes & 50 \\
\hline 21 & $\mathrm{~L}$ retroperitoneum & 23 & No & Liposarcoma & Low & No & No & Yes & 50 \\
\hline 22 & L posterior shoulder & 49 & Yes & Pleomorphic sarcoma & High & Yes & Yes & No & 0 \\
\hline 23 & $\mathrm{~L}$ retroperitoneum & 73 & No & $\begin{array}{l}\text { Malignant fibrous } \\
\text { histiocytoma }\end{array}$ & High & No & No & Yes & 54 \\
\hline 24 & $\begin{array}{l}\mathrm{R} \text { cervical paraspinal } \\
\text { region }\end{array}$ & 77 & No & Peripheral nerve sheath & High & No & No & No & 0 \\
\hline 25 & $\mathrm{~L}$ retroperitoneum & 78 & No & $\begin{array}{l}\text { Hemangiopericytoma/c } \\
\text { lear cell }\end{array}$ & Low & No & No & No & 0 \\
\hline
\end{tabular}

Note. $\mathrm{R}=$ Right, $\mathrm{L}=\mathrm{Left}, \mathrm{RT}=$ Radiation, $\mathrm{Gy}=$ Gray

\subsection{Treatment details}

Sixty-four percent of tumors were treated with neoadjuvant treatment, $48 \%(\mathrm{n}=12)$ with radiation and $8 \%$ chemotherapy $(n=2)$ with 1 patient undergoing both. Median preoperative radiation dose was 50Gy (range 45-54). Preoperative chemotherapy included Mesna, Adriamycin, and Ifosfamide (MAI) in 2 patients and Gemcitabine and Docetaxel in one patient.

Sites of IORT treatment varied based on location of the tumor and adjacent at risk normal tissues and included 17 bone, 4 joint, 6 soft tissue, 2 neurovascular, 1 nerve and 1 pancreas. IORT treatment details, complications and recurrences can be seen in Table 3. One tumor was treated at 2 separate sites (iliofemoral vessels and hip joint). Median IORT dose was $12 \mathrm{~Gy}$ (range 10-17.5), most often prescribed to a depth of $5 \mathrm{~mm}$ from the surface of the flap (range 0-7.5). 
Table 3. IORT Treatment Details, Complications and Outcomes by Tumor

\begin{tabular}{|c|c|c|c|c|c|c|c|c|c|c|c|c|c|}
\hline Tumor & $\begin{array}{l}\text { Area of } \\
\text { concern }\end{array}$ & $\begin{array}{l}\text { Dose } \\
\text { (Gy) }\end{array}$ & $\begin{array}{l}\text { Prescribed } \\
\text { tx. Depth } \\
(\mathbf{c m})\end{array}$ & $\begin{array}{l}\# \\
\text { Cath. } \\
\text { Placed }\end{array}$ & $\begin{array}{l}\# \\
\text { Cath. } \\
\text { Used }\end{array}$ & $\begin{array}{l}\text { Catheter } \\
\text { Length } \\
\text { (cm) }\end{array}$ & $\begin{array}{l}\text { Defect } \\
\text { Size }\end{array}$ & $\begin{array}{l}\text { Adjacent } \\
\text { Structures } \\
\text { Shielded }\end{array}$ & Compl. & $\begin{array}{l}\text { Compl. } \\
\text { Grade }\end{array}$ & $\begin{array}{l}\text { Compl. } \\
\text { treatments }\end{array}$ & $\begin{array}{l}\text { R1 } \\
\text { Margin }\end{array}$ & $\begin{array}{l}\text { Recurren } \\
\text { ce }\end{array}$ \\
\hline 2 & Tibia & 10 & Surface & 7 & 6 & $7 \times 5 ; 8 \times 3$ & $6 \times 7$ & $\begin{array}{l}\text { Skin, soft } \\
\text { tissue }\end{array}$ & Drainage & 1 & Packing & No & $\mathrm{N}$ \\
\hline 3 & Femur & 10 & Surface & 1 & 1 & 7 & NA & $\begin{array}{l}\text { Skin, soft } \\
\text { tissue }\end{array}$ & 0 & 0 & NA & No & Distant \\
\hline 4 & Tibia & 10 & Surface & 3 & 3 & 11 & $3 \times 11$ & $\begin{array}{l}\text { Skin, soft } \\
\text { tissue }\end{array}$ & $\begin{array}{l}\text { Wound } \\
\text { dehiscence }\end{array}$ & $3 b$ & $\begin{array}{l}\text { Debridement } \\
\text { in OR }\end{array}$ & No & $\mathrm{N}$ \\
\hline 5 & $\begin{array}{l}\text { Fascia; } \\
\text { Neuro-va } \\
\text { scular }\end{array}$ & 10 & Surface & 4 & 4 & $\begin{array}{l}4 \mathrm{~cm}+14 \\
\mathrm{~cm} \text { gap }+ \\
4 \mathrm{~cm}\end{array}$ & 4 & Nerve & $\begin{array}{l}\text { Dehiscence } \\
\text { cellulitis }\end{array}$ & 1 & NA & Yes & $\mathrm{N}$ \\
\hline 6 & Tibia & 10 & Surface & 5 & 5 & $8,10 \times 3,9$ & $11 \times 5$ & & $\begin{array}{l}\text { Necrosis, } \\
\text { wound } \\
\text { dehiscence, } \\
\text { cellulitis }\end{array}$ & $3 b$ & OR for flap & No & $\mathrm{N}$ \\
\hline 7 & $\begin{array}{l}\text { Sacral } \\
\text { Promonto } \\
\text { ry }\end{array}$ & 10 & 0.5 & 4 & 4 & $4 \mathrm{~cm}$ & $4 \times 4$ & $\begin{array}{l}\text { Vascular } \\
\text { graft, } \\
\text { intra-abdomi } \\
\text { nal organs, } \\
\text { skin }\end{array}$ & 0 & 0 & NA & No & Distant \\
\hline 8 & Femur & 12.5 & 0.5 & 10 & 10 & $20 \mathrm{~cm}$ & $20 \times 10$ & & 0 & 0 & NA & No & Distant \\
\hline 9 & $\begin{array}{l}\text { Ileofemor } \\
\text { al } \\
\text { Vessels; } \\
\text { Hip Joint }\end{array}$ & 10 & 0.5 & 5 & 5 & 6 & $5 \times 7$ & & Cellulitis & 2 & $\begin{array}{l}\text { Readmitted } \\
\text { for abx }\end{array}$ & No & Distant \\
\hline 10 & $\begin{array}{l}\text { Bony } \\
\text { Spine }\end{array}$ & 17.5 & 0.5 & 6 & 6 & 12 & NA & & 0 & 0 & NA & No & $\mathrm{N}$ \\
\hline 11 & Pancreas & 10 & 0.3 & 4 & 4 & $12 \mathrm{~cm}$ & NA & & Cellulitis & 1 & Wound care & Yes & $\mathrm{N}$ \\
\hline 12 & $\begin{array}{l}\text { Bony } \\
\text { Spine }\end{array}$ & 15 & 0.5 & 8 & 8 & 18 & $8 \times 18$ & $\begin{array}{l}\text { Ligament of } \\
\text { Treitz, aorta, } \\
\text { iliac artery, } \\
\text { etc }\end{array}$ & $\begin{array}{l}\text { Cellulitis, } \\
\text { Abscess, } \\
\text { fistula }\end{array}$ & $3 b$ & $\begin{array}{l}\text { Abx, OR for } \\
\text { washout and } \\
\text { colostomy }\end{array}$ & No & $\mathrm{N}$ \\
\hline 13 & $\begin{array}{l}\text { Bony } \\
\text { Spine }\end{array}$ & 12.5 & 0.5 & 3 & 3 & 10 & $9 \times 3$ & $\begin{array}{l}\text { Bowel, } \\
\text { pancreas, } \\
\text { spleen and } \\
\text { skin }\end{array}$ & 0 & 0 & 0 & No & Distant \\
\hline 14 & $\begin{array}{l}\text { Bony } \\
\text { Spine }\end{array}$ & 15 & 0.5 & 8 & 8 & $\begin{array}{l}6.5 \mathrm{~cm} \times 4, \\
12 \mathrm{~cm} \times 4\end{array}$ & $\begin{array}{l}8 \times 12 \\
\text { and } 8 \times \\
7\end{array}$ & $\begin{array}{l}\text { Dura, spinal } \\
\text { cord, } \\
\text { esophagus, } \\
\text { aorta, } \\
\text { pericardium, } \\
\text { heart, soft } \\
\text { tissue, skin }\end{array}$ & $\begin{array}{l}\text { Chyle leak, } \\
\text { cardiopulm } \\
\text {-onary } \\
\text { instability }\end{array}$ & $4 \mathrm{~b}$ & $\begin{array}{l}\text { OR } \\
\text { Debridement } \\
\text { /mesh } \\
\text { removal, } \\
\text { ligation } \\
\text { thoracic duct }\end{array}$ & No & $\mathrm{N}$ \\
\hline 15 & $\begin{array}{l}\text { R Chest } \\
\text { Wall, } \\
\text { Axillary } \\
\text { Vein }\end{array}$ & 6.25 & 0.75 & 6 & 6 & $7 \mathrm{~cm}$ & $6 \times 7$ & NA & $\begin{array}{l}\text { Erythema, } \\
\text { joint pain }\end{array}$ & 2 & $\begin{array}{l}\text { Abx, joint } \\
\text { injections }\end{array}$ & No & Distant \\
\hline 16 & $\begin{array}{l}\text { L Pelvic } \\
\text { Floor }\end{array}$ & 12.5 & 0.5 & 10 & 10 & $16 \mathrm{~cm}$ & $10 \times 16$ & $\begin{array}{l}\text { L kidney, } \\
\text { ureter, distal } \\
\text { femoral } \\
\text { nerve, small } \\
\text { bowel, and } \\
\text { skin }\end{array}$ & $\begin{array}{l}\text { Wound } \\
\text { infection }\end{array}$ & 2 & $\begin{array}{l}\text { Abx, wound } \\
\text { care }\end{array}$ & No & Distant \\
\hline 17 & $\begin{array}{l}\text { Vertebral } \\
\text { Foramina }\end{array}$ & 7.5 & 0.5 & 6 & 6 & $16 \mathrm{~cm}$ & $6 \times 16$ & L kidney & $\begin{array}{l}\text { Bilateral } \\
\text { lower } \\
\text { extremity } \\
\text { weakness, } \\
\text { incontinenc } \\
\text { e }\end{array}$ & 1 & NA & Yes & $\mathrm{N}$ \\
\hline 18 & $\begin{array}{l}\text { Vertebral } \\
\text { Body }\end{array}$ & 12.5 & 0.5 & 7 & 7 & $9 \mathrm{~cm}$ & $7 \times 9$ & $\begin{array}{l}\text { R kidney, } \\
\text { duodenum, } \\
\text { inferior vena } \\
\text { cava, R iliac } \\
\text { vessels, } \\
\text { ureter, } \\
\text { subcutaneou } \\
\text { s tissue }\end{array}$ & $\begin{array}{l}\mathrm{R} \text { leg } \\
\text { weakness }\end{array}$ & 1 & NA & No & Distant \\
\hline
\end{tabular}


Table 3. (Continued.)

\begin{tabular}{|c|c|c|c|c|c|c|c|c|c|c|c|c|c|}
\hline Tumor & $\begin{array}{l}\text { Area of } \\
\text { concern }\end{array}$ & $\begin{array}{l}\text { Dose } \\
\text { (Gy) }\end{array}$ & $\begin{array}{l}\text { Prescribed } \\
\text { tx. Depth } \\
\text { (cm) }\end{array}$ & $\begin{array}{l}\text { \# } \\
\text { Cath. } \\
\text { Placed }\end{array}$ & $\begin{array}{l}\# \\
\text { Cath. } \\
\text { Used }\end{array}$ & $\begin{array}{l}\text { Catheter } \\
\text { Length } \\
\text { (cm) }\end{array}$ & $\begin{array}{l}\text { Defect } \\
\text { Size }\end{array}$ & $\begin{array}{l}\text { Adjacent } \\
\text { Structures } \\
\text { Shielded }\end{array}$ & Compl. & $\begin{array}{l}\text { Compl. } \\
\text { Grade }\end{array}$ & $\begin{array}{l}\text { Compl. } \\
\text { treatments }\end{array}$ & $\begin{array}{l}\text { R1 } \\
\text { Margin }\end{array}$ & $\begin{array}{l}\text { Recurren } \\
\text { ce }\end{array}$ \\
\hline 20 & $\begin{array}{l}\text { Glenohu } \\
\text { meral } \\
\text { Joint }\end{array}$ & 15 & 0.5 & 7 & 7 & $4.5 \mathrm{~cm}$ & $7 \times 6$ & NA & $\begin{array}{l}\text { Lymph-ede } \\
\text { ma }\end{array}$ & 1 & NA & No & $\mathrm{N}$ \\
\hline 21 & $\begin{array}{l}\text { L Bony } \\
\text { Spine, } \\
\text { Retroperit } \\
\text { oneum }\end{array}$ & 10 & 0.5 & 5 & 5 & $4.5 \mathrm{~cm}$ & NA & NA & Ascites & 3a & Paracentesis & Yes & $\mathrm{N}$ \\
\hline 22 & $\begin{array}{l}\text { Joint } \\
\text { Capsule }\end{array}$ & 12 & 0.5 & 6 & 6 & $5 \mathrm{~cm}$ & $6 \times 5$ & NA & 0 & 0 & NA & No & Local \\
\hline 24 & $\begin{array}{l}\text { C2 } \\
\text { Verterbra } \\
\text { l Body }\end{array}$ & 15 & Surface & 4 & 4 & $\begin{array}{l}6 \mathrm{~cm} * 1, \\
8 \mathrm{~cm} * 3\end{array}$ & $4 \times 8$ & $\begin{array}{l}\text { Trachea, } \\
\text { esophagus }\end{array}$ & $\begin{array}{l}\text { Fascial } \\
\text { cellulitis }\end{array}$ & 2 & Readmission & No & $\mathrm{N}$ \\
\hline 25 & $\begin{array}{l}\text { Psoas, } \\
\text { Ribs }\end{array}$ & 12.5 & Surface & 11 & 11 & $21[\max ]$ & NA & $\begin{array}{l}\text { Small bowel, } \\
\text { kidney }\end{array}$ & & 4a & $\begin{array}{l}\text { Prolonged } \\
\text { intubation }\end{array}$ & No & $\mathrm{N}$ \\
\hline
\end{tabular}

Post-resection tumor sites were closed primarily in 60\%. Complex closures included 1 mesh for abdominal wall reconstruction, 6 advancement/rotational flaps, 2 advancement/rotational flaps in combination with a split-thickness skin graft, and 1 reconstruction with a prosthetic device (spine). Median estimated blood loss was 300ml (5-2000). When stratifying by location, we observed significant associations with estimated blood loss. Extremity sarcomas had a median of $30 \mathrm{ml}$ (5-300) versus retroperitoneal sarcomas with a median of 500ml $(300-1700)$ of blood loss $(p<0.001)$.

Margins were microscopically positive in $20 \%(n=5)$. No resections had macroscopically positive margins. Sites of margin positivity correlated with areas pre-operatively suspected to be at risk and included: pancreas, paraspinal/vertebral foramina, bony spine, left hemidiaphragm and fascial/neurovascular bundle of the lower extremity. Five patients had tumor within $2 \mathrm{~mm}$ of the inked margin. When stratifying for margin status, significant associations were observed with tumor grade, with low-grade tumors having higher positive margin status $(p=0.032)$. Median intraoperative radiation dosing for negative resections margins was $12.5 \mathrm{~Gy}$ versus 10Gy in patients with positive margins. When stratifying for positive margin status, no increases were found for either local or distant recurrences ( $p=1.00$ and 0.62 respectively). Lower extremities tumors received a median of $10.3 \mathrm{~Gy}$ versus $13.1 \mathrm{~Gy}$ for upper extremities $(p=0.013)$. When stratifying for location with extremity versus retroperitoneal tumors, no significant differences were found for local or distant recurrences ( $p=0.48$ versus 0.67 respectively) in this patient cohort.

\subsection{Treatment results}

Any postoperative morbidity occurred in $72 \%$, but complications potentially attributable to IORT at the time of resection were infection $(n=8)$, delayed wound healing $(n=3)$, neuropathy $(n=2)$, and GI fistula $(n=1)$. High-grade complications occurred in 33.3\%, for which 4 patients required reoperation and 5 required readmission. Perioperative mortality was $0 \%$.

\subsection{Adjuvant treatment}

Five tumor sites received postoperative radiation at a median dose of 46Gy (45-50). Three tumors were treated postoperative with chemotherapy, one of which had radiation as well (Gemcitabine/Docetaxel in 2; unknown type in 1). 


\subsection{Follow up and survival}

At a median follow-up of 20.1 months (range 2.7-96.4), 2-year LR free survival was 60.8\%, distant metastasis free survival was $38.4 \%$ and disease specific survival was $84.0 \%$.

Two year LRFS was $60.8 \%$, distant metastasis free survival was $50.1 \%$, and disease specific survival was $84.0 \%$ (see Table 4 and Figure 2 and 3). Overall median survival was 42.2 months and 70.5\% at 2 years. Median DFS was 13.7 months and $28.9 \%$ at 2 years. No recurrences occurred at the site of IORT. Two tumors recurred within the prior resection fields as well as prior EBRT. One patient had a history of EBRT prior to resection and the other was treated with resection and IORT followed by EBRT but recurred locally (not at site of IORT). When stratified by local recurrence and complication status, no statistically significant associations were found.

Table 4. Two-year survivals for all patients treated with IORT at the time of resection

\begin{tabular}{ll}
\hline 2 Year Survival & \% \\
\hline Local Recurrence Free & 60.8 \\
Distant Metastasis Free & 38.4 \\
Disease Free & 28.9 \\
Disease Specific & 84.0 \\
Overall & 70.5 \\
\hline
\end{tabular}

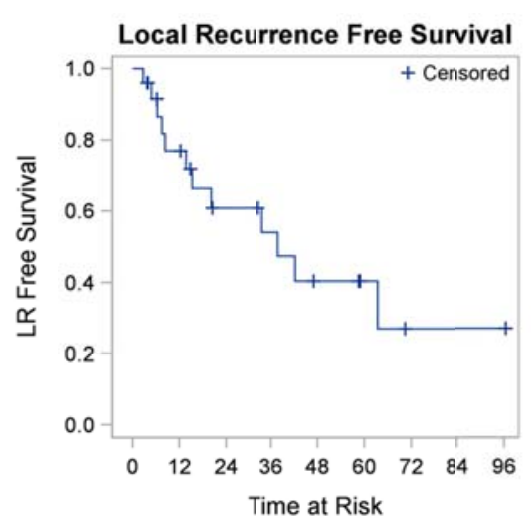

Figure 2. Local Recurrence Free for all Patients Treated with IORT in Months. LR= local recurrence.

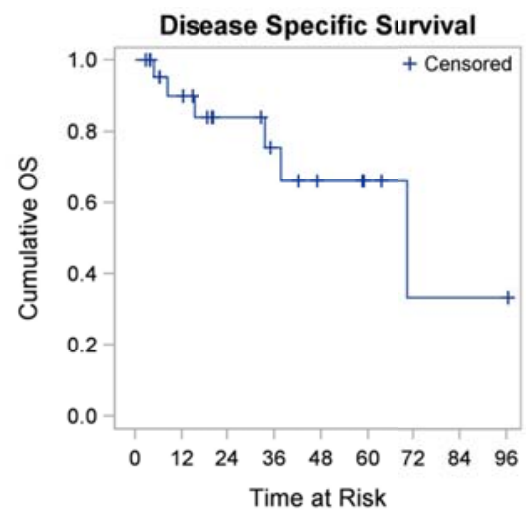

Figure 3. Disease Specific Survival for all Patients Treated with IORT in Months. OS= overall survival.

\section{Discussion}

In this study we showed that IORT is a safe addition to resection and peri-operative external beam radiation in patients at high risk for local recurrence. There were no recurrences at the site of IORT and our outcomes are comparable to prior literature. For retroperitoneal sarcomas (RPS), Stucky et al. showed a 5-year local recurrence rate of 22\% in tumors treated with neoadjuvant RT followed by surgery and IORT, while Sindelar et al. showed an 8-year rate of 40\% with surgery, IORT and adjuvant RT ${ }^{[15,16]}$. While no retroperitoneal tumors recurred locoregionally in our study, longer follow-up would potentially capture recurrences given the median time to local recurrence in prior literature was approximately 21 months ${ }^{[16]}$. At a more comparable median follow-up of 20 months, Dziewirski et al. showed a LRFS of $51 \%$ for retroperitoneal sarcomas, although median time to recurrence was not described ${ }^{[19]}$. At 24 months, our LRFS was $61 \%$ for all tumors (55\% for extremity and $70 \%$ for retroperitoneal). For extremity sarcomas, locoregional control ranged from $78 \%$ to $100 \%$, comparable to our $81 \%$ (2 patients recurred locally) ${ }^{[18,21,22,24-26]}$. When compared to data including only resection and EBRT, local control rates range from 66\%-100\% but have shown to be lower in tumors with positive margins, which includes $20 \%$ of our patients ${ }^{[2,3,9,10,28]}$. 
Surgical resection with negative margins and radiation therapy in appropriate patients remains the standard of care for local control but distant disease control continues to be a problem. These primary treatment modalities of surgical resection and radiation, EBRT and/ IORT when appropriate, are local therapies. At 2 years, our OS was $75 \%$ and DSS was $84 \%$. While we were unable to determine predictors for increased local or distant recurrences, our patients were high risk for any recurrence since $84 \%$ of tumors were intermediate to high grade and all were high risk for positive margins.

These multiple high-risk features in our cohort placed them not only at high risk for local recurrence but also distant metastases. Of patients that developed recurrences, 83\% were distant metastases. Prior literature show an OS rate of $55 \%-60 \%$ for RPS and a DFS rate of $43 \%-84 \%$ and OS rate of $52 \%-78 \%$ for extremity tumors ${ }^{[15,17,21,22,24,29]}$. We found a lower DFS of $28.9 \%$. While $3(12 \%)$ patients were treated with neoadjuvant chemotherapy and $3(12 \%)$ were treated with adjuvant therapy, no difference in distant metastasis occurrences were seen. Some literature does suggest perioperative systemic chemotherapy may improve disease control and even survival but further studies using all treatment modalities are needed to address distant disease control in high-risk patients ${ }^{[29-34]}$.

The NCCN Guidelines Version 2.2014 recommends IORT boost doses of 10-12.5 Gy for microscopic disease and 15 Gy for gross residual disease for tumors of the extremities, head/neck, and retroperitoneum in general ${ }^{[12,35]}$. There are different techniques via which IORT can be delivered, including HDR brachytherapy as used at our institution. We gave a median overall dose of $12 \mathrm{~Gy}$ intraoperatively. The choice of dose and depth for dose prescription was based on target site for treatment, risk for depth of invasion, concern of margin status, and normal adjacent tissues in the treatment field. Examples of dose variability for these reasons include an area adjacent to the bony spine treated to $17.5 \mathrm{~Gy}$ (prescribed to a depth from the flap of $5 \mathrm{~mm}$ ), an area at risk near the axillary vein with concern for deep involvement treated to $6.25 \mathrm{~Gy}$ (prescribed to a treatment depth of $7.5 \mathrm{~mm}$ ) and $7.5 \mathrm{~Gy}$ (depth of treatment $5 \mathrm{~mm}$ ) at the vertebral foramina.

Our study had an overall 68\% complication rate with a $16 \%$ rate of requiring reoperation, and no perioperative mortality. There may be multiple confounding factors contributing to this complication rate. Dziewirski et al. reported a $21.5 \%$ reoperation rate after resection with IORT for RPS with adjuvant EBRT and there was one postoperative death ${ }^{\text {[19] }}$. Rachbauer et al., resulted no morbidity after intraoperative brachytherapy, but their $28 \%$ morbidity occurred after adjuvant EBRT where $21 \%$ required reoperation ${ }^{[22]}$. Llácer et al., in a review of tumors involving the neurovascular bundle, reported a $28 \%$ acute morbidity where $12.7 \%$ of patients required reoperation and $44 \%$ suffered a late morbidity ${ }^{[23]}$. An increased risk of neuropathy (up to 60\%) has also been shown for RPS with IORT ${ }^{[16]}$. In our study, 4 (16\%) required reoperation and neuropathy occurred in 2 (8\%) patients, both with RPS. Our overall wound complication rate was 32\% and infections were our most common complication. Preoperative radiation has been shown to adversely affect wound healing ${ }^{[7,36]}$. Fourteen patients (56\%) had neoadjuvant radiation and $3(12 \%)$ more, all presenting with locally recurrent tumors, had a history of prior radiation at the site of resection, likely attributing to an increased wound complication rate.

While future studies are necessary to address distant disease control and survival, IORT can be used to deliver a radiation boost during resection to areas at high risk for recurrence when critical structures preclude wide resection margins. Currently accruing is the German Clinical Phase I/II trial on neodjuvant IMRT and IORT for retroperitoneal sarcomas ${ }^{[20]}$. Until further data is available, IORT at the time of resection can be an additional treatment modality in specialized STS centers to improve local tumor control without significantly increased complications.

\section{Conclusion}

IORT at the time of STS resection was effective in preventing LR at the treated site, despite multiple high risk features for recurrence. Further studies to improve outcomes including local control and DSS are needed.

\section{Competing interests}

We have nothing to disclose.

Published by Sciedu Press 


\section{References}

[1] Al-Absi E, Farrokhyar F, Sharma R, et al. A systematic review and meta-analysis of oncologic outcomes of pre- versus postoperative radiation in localized resectable soft-tissue sarcoma. Ann Surg Oncol. 2010; 17(5): 1367-1374. Accessed 12 June 2014. http://dx.doi.org/10.1245/s10434-009-0885-7

[2] Beane JD, Yang JC, White D, et al. Efficacy of adjuvant radiation therapy in the treatment of soft tissue sarcoma of the extremity: 20-year follow-up of a randomized prospective trial. Ann Surg Oncol. 2014. Accessed 12 June 2014.

http://dx.doi.org/10.1245/s10434-014-3732-4

[3] Delaney TF, Liebsch NJ, Pedlow FX, et al. Long-term results of phase II study of high dose photon/proton radiotherapy in the management of spine chordomas, chondrosarcomas, and other sarcomas. J Surg Oncol. 2014. http://dx.doi.org/10.1002/jso.23617

[4] Rosenberg SA, Tepper J, Glatstein E, et al. The treatment of soft-tissue sarcomas of the extremities. Prospective randomized evaluations of (1) limb-sparing surgery plus radiation therapy compared with amputation and (2) the role of adjuvant chemotherapy. Ann Surg. 1982; 196(3): 305-315. Accessed 12 June 2014. http://dx.doi.org/10.1097/00000658-198209000-00009

[5] Tuan J, Vitolo V, Vischioni B, et al. Radiation therapy for retroperitoneal sarcoma. Radiol Med. 2014. Accessed 12 June 2014. http://dx.doi.org/10.1007/s11547-013-0350-3

[6] Yang JC, Chang AE, Baker AR, et al. Randomized prospective study of the benefit of adjuvant radiation therapy in the treatment of soft tssue sarcomas of the extremity. J Clin Oncol. 1998; 16(1):197-203. Accessed 12 June 2014. PMid:9440743

[7] O'Sullivan B, Davis AM, Turcotte R, et al. Preoperative versus postoperative radiotherapy in soft-tissue sarcoma of the limbs: A randomised trial. Lancet. 2002; 359(9325):2235-2241. http://dx.doi.org/10.1016/S0140-6736(02)09292-9

[8] Alektiar KM, Velasco J, Zelefsky MJ, et al. Adjuvant radiotherapy for margin-positive high-grade soft tissue sarcoma of the extremity. Int J Radiat Oncol Biol Phys. 2000; 48(4): 1051-1058. Accessed 12 June 2014.

http://dx.doi.org/10.1016/S0360-3016(00)00753-7

[9] DeLaney TF, Kepka L, Goldberg SI, et al. Radiation therapy for control of soft-tissue sarcomas resected with positive margins. Int J Radiat Oncol Biol Phys. 2007; 67(5): 1460-1469. Accessed 12 June 2014. http://dx.doi.org/10.1016/j.ijrobp.2006.11.035

[10] Sadoski C, Suit HD, Rosenberg A, et al. Preoperative radiation, surgical margins, and local control of extremity sarcomas of soft tissues. J Surg Oncol. 1993; 52(4): 223-230. Accessed 12 June 2014.

[11] Sole CV, Calvo FA, de Sierra PA, et al. Multidisciplinary therapy for patients with locally oligo-recurrent pelvic malignancies. J Cancer Res Clin Oncol. 2014. Accessed 12 June 2014. http://dx.doi.org/10.1007/s00432-014-1667-6

[12] DeLaney TF, Trofimov AV, Engelsman M, Suit HD. Advanced-technology radiation therapy in the management of bone and soft tissue sarcomas. Cancer Control. 2005; 12(1): 27-35. Accessed 12 June 2014.

[13] Brennan MF, Hilaris B, Shiu MH, et al. Local recurrence in adult soft-tissue sarcoma. A randomized trial of brachytherapy. Arch Surg. 1987; 122(11): 1289-1293. Accessed 12 June 2014. PMid:3314794

[14] Alektiar KM, Brennan MF, Healey JH, Singer S. Impact of intensity-modulated radiation therapy on local control in primary soft-tissue sarcoma of the extremity. J Clin Oncol. 2008; 26(20): 3440-3444. Accessed 12 June 2014. doi:http://dx.doi.org/10.1200/JCO.2008.16.6249

[15] Stucky C-H, Wasif N, Ashman JB, et al. Excellent local control with preoperative radiation therapy, surgical resection, and intra-operative electron radiation therapy for retroperitoneal sarcoma. J Surg Oncol. 2014; 109(8): 798-803. Accessed 12 June 2014. http://dx.doi.org/10.1002/jso.23576

[16] Sindelar WF, Kinsella TJ, Chen PW, et al. Intraoperative radiotherapy in retroperitoneal sarcomas: Final results of a prospective, randomized, clinical trial. Arch Surg. 1993; 128(4): 402-410. Accessed 12 June 2014. http://dx.doi.org/10.1001/archsurg.1993.01420160040005

[17] Calvo FA, Sole CV, Cambeiro M, et al. Prognostic value of external beam radiation therapy in patients treated with surgical resection and intraoperative electron beam radiation therapy for locally recurrent soft tissue sarcoma: A multicentric long-term outcome analysis. Int J Radiat Oncol Biol Phys. 2014; 88(1): 143-150. Accessed 12 June 2014. http://dx.doi.org/10.1016/j.ijrobp.2013.10.021

[18] Calvo FA, Sole CV, Polo A, et al. Limb-sparing management with surgical resection, external-beam and intraoperative electron-beam radiation therapy boost for patients with primary soft tissue sarcoma of the extremity: A multicentric pooled analysis of long-term outcomes. Strahlenther Onkol. 2014. http://dx.doi.org/10.1007/s00066-014-0640-2

[19] Dziewirski W, Rutkowski P, Nowecki ZI, et al. Surgery combined with intraoperative brachytherapy in the treatment of retroperitoneal sarcomas. Ann Surg Oncol. 2006; 13(2): 245-252. Accessed 12 June 2014.

http://dx.doi.org/10.1245/ASO.2006.03.026 
[20] Roeder F, Schulz-Ertner D, Nikoghosyan AV, et al. A clinical phase I/II trial to investigate preoperative dose-escalated intensity-modulated radiation therapy (IMRT) and intraoperative radiation therapy (IORT) in patients with retroperitoneal soft tissue sarcoma. BMC Cancer. 2012; 12: 287-2407-12-287.

[21] Tran QNH, Kim AC, Gottschalk AR, et al. Clinical outcomes of intraoperative radiation therapy for extremity sarcomas. Sarcoma. 2006. Accessed 12 June 2014.

[22] Rachbauer F, Sztankay A, Kreczy A, et al. High-dose-rate intraoperative brachytherapy (IOHDR) using flab technique in the treatment of soft tissue sarcomas. Strahlenther Onkol. 2003; 179(7): 480-485. PMid:12835885

[23] Llácer C, Delannes M, Minsat M, et al. Low-dose intraoperative brachytherapy in soft tissue sarcomas involving neurovascular structure. Radiother Oncol. 2006; 78(1): 10-16. Accessed 12 June 2014. http://dx.doi.org/10.1016/j.radonc.2005.12.002

[24] Call JA, Stafford SL, Petersen IA, Haddock MG. Use of intraoperative radiotherapy for upper-extremity soft-tissue sarcomas: Analysis of disease outcomes and toxicity. Am J Clin Oncol Cancer Clin Trials. 2014; 37(1): 81-85. Accessed 12 June 2014. http://dx.doi.org/10.1097/COC.0b013e31826b9b3d

[25] Azinovic I, Monge RM, Aristu JJ, et al. Intraoperative radiotherapy electron boost followed by moderate doses of external beam radiotherapy in resected soft-tissue sarcoma of the extremities. Radiother Oncol. 2003; 67(3): 331-337. Accessed 12 June 2014. http://dx.doi.org/10.1016/S0167-8140(03)00163-4

[26] Oertel S, Treiber M, Zahlten-Hinguranage A, et al. Intraoperative electron boost radiation followed by moderate doses of external beam radiotherapy in limb-sparing treatment of patients with extremity soft-tissue sarcoma. Int J Radiat Oncol Biol Phys. 2006; 64(5): 1416-1423. Accessed 12 June 2014. http://dx.doi.org/10.1016/j.ijrobp.2005.10.009

[27] Clavien PA, Barkun J, de Oliveira ML, et al. The clavien-dindo classification of surgical complications: Five-year experience. Ann Surg. 2009; 250(2): 187-196. http://dx.doi.org/10.1097/SLA.0b013e3181b13ca2

[28] Wilson AN, Davis A, Bell RS, et al. Local control of soft tissue sarcoma of the extremity: The experience of a multidisciplinary sarcoma group with definitive surgery and radiotherapy. Eur J Cancer Part A Gen Top. 1994; 30(6):746-751. Accessed 12 June 2014. http://dx.doi.org/10.1016/0959-8049(94)90286-0

[29] Grobmyer SR, Maki RG, Demetri GD, et al. Neo-adjuvant chemotherapy for primary high-grade extremity soft tissue sarcoma. Ann Oncol. 2004; 15(11): 1667-1672. Accessed 12 June 2014. http://dx.doi.org/10.1093/annonc/mdh431

[30] DeLaney TF, Spiro IJ, Suit HD, et al. Neoadjuvant chemotherapy and radiotherapy for large extremity soft-tissue sarcomas. Int J Radiat Oncol Biol Phys. 2003; 56(4): 1117-1127. Accessed 12 June 2014. http://dx.doi.org/10.1016/S0360-3016(03)00186-X

[31] Kraybill WG, Harris J, Spiro IJ, et al. Long-term results of a phase 2 study of neoadjuvant chemotherapy and radiotherapy in the management of high-risk, high-grade, soft tissue sarcomas of the extremities and body wall: Radiation therapy oncology group trial 9514. Cancer. 2010; 116(19): 4613-4621. Accessed 12 June 2014. http://dx.doi.org/10.1002/cncr.25350

[32] Kraybill WG, Harris J, Spiro IJ, et al. Phase II study of neoadjuvant chemotherapy and radiation therapy in the management of high-risk, high-grade, soft tissue sarcomas of the extremities and body wall: Radiation therapy oncology group trial 9514. J Clin Oncol. 2006; 24(4): 619-625. Accessed 12 June 2014. http://dx.doi.org/10.1002/cncr.25350

[33] Mullen JT, Kobayashi W, Wang JJ, et al. Long-term follow-up of patients treated with neoadjuvant chemotherapy and radiotherapy for large, extremity soft tissue sarcomas. Cancer. 2012; 118(15): 3758-3765. Accessed 12 June 2014. http://dx.doi.org/10.1002/cncr.26696

[34] Pervaiz N, Colterjohn N, Farrokhyar F, et al. A systematic meta-analysis of randomized controlled trials of adjuvant chemotherapy for localized resectable soft-tissue sarcoma. Cancer. 2008; 113(3): 573-581. Accessed 12 June 2014.

http://dx.doi.org/10.1002/cncr.23592

[35] National Comprehensive Cancer Network. Soft tissue sarcoma. nccn.org. Updated 2014. Accessed 06/2, 2014.

[36] Davis AM, O'Sullivan B, Bell RS, et al. Function and health status outcomes in a randomized trial comparing preoperative and postoperative radiotherapy in extremity soft tissue sarcoma. J Clin Oncol. 2002; 20(22): 4472-4477. Accessed 12 June 2014. http://dx.doi.org/10.1200/JCO.2002.03.084 